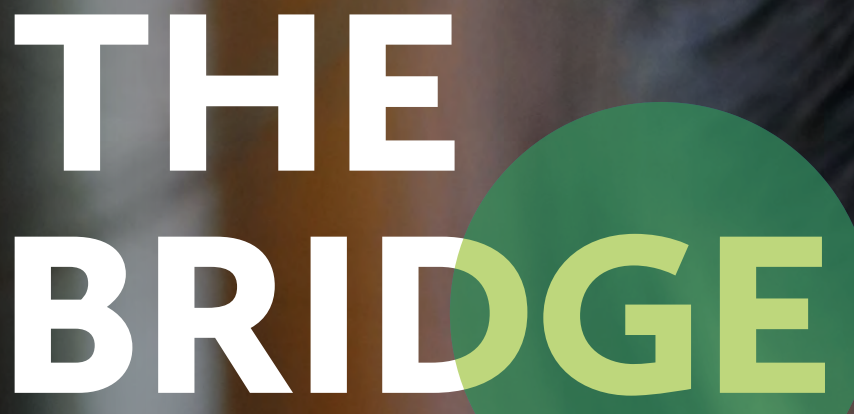

May 2020

\title{
Deafness in Children
}

\section{Can literacy} interventions benefit mental health in children with permanent hearing loss?

\section{Plus}

\section{Research} digests from JCPP and CAMH 


\section{Contents:}

Accessing good communication

- Deaf children in a mental health assessment

Mental Health in Deaf Children

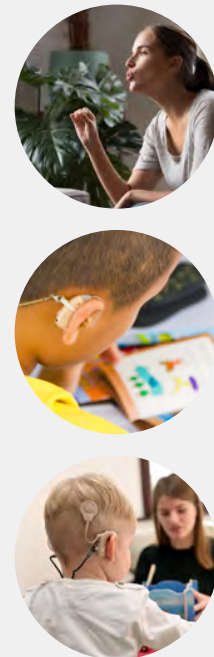

Can literacy interventions benefit mental health in children with permanent hearing loss?

Do audiological classification systems cause more confusion than clarity?

Parents provide their perspective on the crossroads of autism and deafness

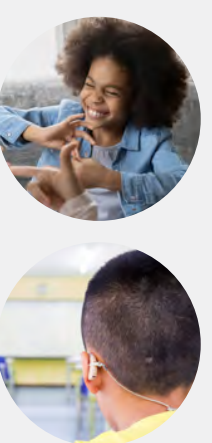

Integrating interpreters into CAMHS: useful tips for effective co-working

Multi-disciplinary teams are needed to sensitively diagnose autism in Deaf children

\section{Clarification}

The current Covid-19 situation has enhanced the need to carry out psychological therapies remotely with families, including pointers to self-help. Self-help and guided-self-help for children and adolescents has been the subject of several research studies. The Bridge wants to remind readers that an article in the last edition 'Online CBT is ineffective for treating adolescent anxiety' (https://www.acamh.org/researchdigest/online-cbt-is-ineffective-for-treating-adolescent-anxiety/hyperlink to Jessica's summary) was a summary of a single important article, and the title was not intended to represent the overall status of the efficacy of online anxiety treatments in youth. There are well evaluated and effective online and other remote interventions for a range of conditions - including anxiety - in children and young people. The readers might want to read a summary published in an earlier issue of The Bridge- https://www.acamh. org/research-digest/meta-analysis-self-help-intervention/ 


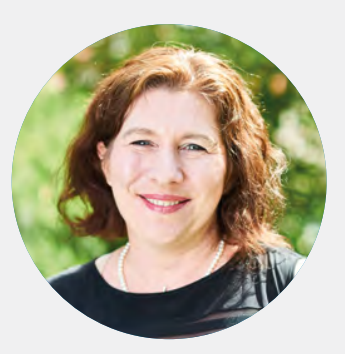

\section{Dr Juliette Kennedy}

\section{The Bridge Editor}

I am Dr. Juliette Kennedy, Editor of The Bridge, and a Consultant Child and Adolescent Psychiatrist working clinically in a North Yorkshire CAMHS team. I am Associate Director of Medical Education in the trust I work in, also Training Program director for CAMHS higher training in Yorkshire.

The Bridge presents the most clinically-relevant research from our two peer-reviewed journals: Child and Adolescent Mental Health and The Journal of Child Psychology and Psychiatry, as well as interesting and important studies from the wider literature. Please let us know what you'd like to see in upcoming editions by sending an email to me at: researchdigests@acamh.org

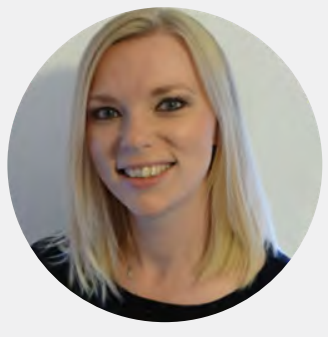

\section{Dr Jessica K. Edwards}

Research highlights in this edition are prepared by Dr Jessica K. Edwards. Jessica is a freelance editor and science writer, and started writing for 'The Bridge' in December 2017.

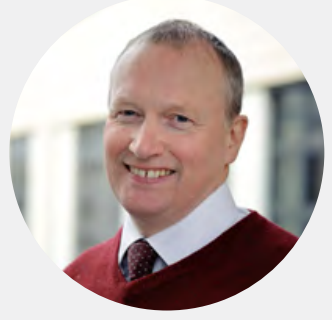

\section{Professor Barry Wright}

Barry Wright is a Professor of Child Mental Health at the University of York working for the Hull York Medical School and the Leeds and York Partnership NHS Foundation Trust. $\mathrm{He}$ is Clinical Lead of the National Deaf CAMHS, which is a specialist service for deaf children and young people run in ten centres across England and funded by the NHS England. This has Deaf and hearing clinicians working together to deliver accessible therapies to D/deaf children and young people across England. He runs a research group called the Child Oriented Mental health Intervention Centre (COMIC), which focuses its attention on finding and researching child friendly mental health interventions that can be delivered at scale in the community (https://www.comic.org.uk/research) 



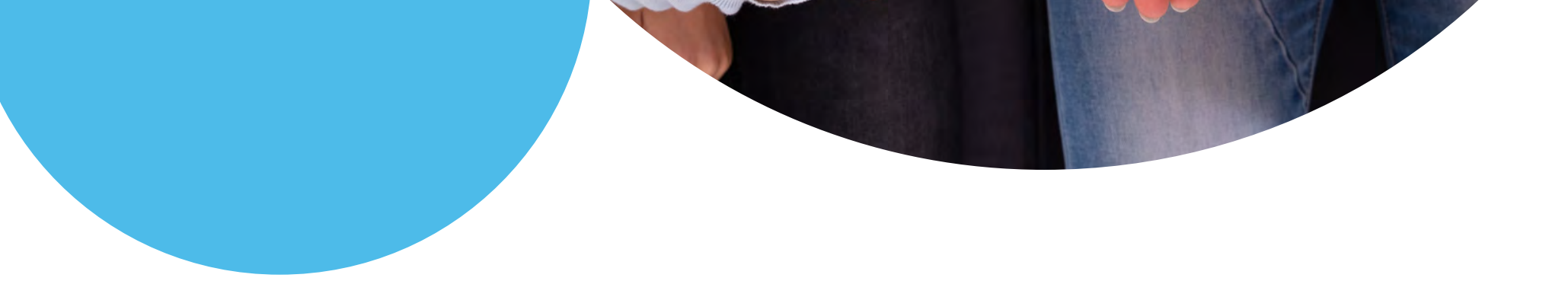

If we focus on assessments for deaf people whose first language is sign language then we can see just how important this may become. In the UK BSL became a recognised language in 2003, and one might assume from this that attaining an assessment in one of the land's recognised languages would be an acceptable thing to expect. However, most deaf people attending primary care for an appointment described poor communication usually without the benefit of a qualified sign language interpreter or similar (Emond et al, 2015).

There is surprisingly little written about this issue until recently. Working alongside deaf clinical colleagues Vicci Ackroyd, who runs the interpreting services in the Northern arm of the National Deaf Child and Adolescent Mental Health Service (NDCAMHS), laid out a set of straightforward principles for good practice (Ackroyd et al 2018) - see box 1. These are now usual practice in the 10 centre NHS England funded NDCAMHS service across the country. For people arriving in GP practices or emergency services these are not always readily available and can be dependent on local decisions and funding available. The good news is that there are a growing number of helpful services that can be accessed online. These include services where a deaf person can sign into a computer camera to someone on the end of the videoconference and that person will then speak back to the assessing clinician and then translate into sign language when that clinician responds. BSLHealthAccess is a collaboration between charity SignHealth and InterpreterNow, a social enterprise, who is the provider for some NHS 111 access (see https://www.bslhealthaccess.co.uk/). Other examples include SignLive and Sign Video.

\section{Assessment for ASD as an exemplar}

From conception through to childhood our brain and nervous systems are developing. Neurodevelopmentally we are changing all the time and these changes are not just responsive to our genetic coding, but also highly responsive to our experiences, interactions, our lifestyle (e.g. sleep, nutrition) and a range of other things that are interacting. Neurodevelopmental difference is therefore complex. As a separate domain from medical diagnosis it presents a number of challenges when we are assessing a child at one specific point of time for the presence or absence of neurodevelopmental differences such as autism spectrum conditions. The assessment conditions also make a difference.

One of the things that we often assess for as part of the assessment process in Autism Spectrum Conditions (ASC) is 'theory of mind'. This is our ability to make guesses about how someone else is thinking or feeling and is one of the markers for ASC. However, even in ASC this set of skills changes as we grow and develop and is influenced by our experiences.

Several pieces of important research have shown that theory of mind and empathy skill delays can occur in a range of conditions other than ASC including children with language impairment (Peterson, 2016; AndrésRoqueta et al, 2013) and children who are neglected in early life (Luke \& Banerjee, 2013). Early life trauma also disrupts the way we pay attention to or respond to the thoughts or feelings of others (Fonagy et al, 2017). We simply don't have enough research yet to fully understand whether the developmental trajectories of these different pathways are different, or whether different interventions may be more or less effective for each group. 
Another group where there is a wealth of international research showing theory of mind delays is a group of deaf children with language deprivation in early life (Peterson, 2016). Signing deaf children in signing families usually do not have these delays (Schick et $\mathrm{al}, 2007)$. This can lead to the misdiagnosis of some deaf children as having autism. Some believe that this is almost indistinguishable from autism whilst others believe that the two sets of circumstances are different with different developmental trajectories and different responses to intervention (Wright \& Oakes, 2012). Further research needs to be done into this important area, because many of these deaf children with social and emotional developmental delay are placed in education autism units. Given that they already have communication difficulties as a result of being deaf, then placing such children in an autism unit may restrict their potential for the rich experiences needed for social development.

Recent research shows that families seeking an autism assessment for their deaf child often run into a range of problems accessing a good assessment and may experience long delays (Young et al, 2019). Clinicians feel under-skilled and unprepared to carry out such assessments (Brenman et al, 2014). For these reasons the Medical Research Council has funded a long piece of research to improve the autism assessment instruments for deaf children to include a screening instrument, a parent semi-structured interview and a play/interaction based assessment. This research will be reporting these important results to its 5 year study in $2020 / 21$. Hopefully, this will lead to the important further research that is necessary to better understand the needs of deaf children with autism. This will also help to characterise children more clearly in order to be able to generate better interventions and better educational placements for them.

Experiences of parenting deaf children more generally shows just why specialised services are needed (Beresford et al, 2008). Deaf children requiring services should of course have access to generic services as usual, but they need access to deaf clinicians for playbased assessments and access to qualified interpreters if their first language is BSL.
Box 1: Advice to generic CAMHS clinicians carrying out an assessment with a deaf child or parent whose first language is British Sign Language

Reproduced by kind permission of Vicci Ackroyd from Figure 2 in Ackroyd, V. \& Wright, B. (2018), Working with British Sign Language (BSL) interpreters: lessons from child and adolescent mental health services in the U.K. J. Commun. Healthc. 11: 195-204. doi: 10.1080/17538068.2018.1492218.

1. Check that the interpreter is qualified, has three years post qualification experience that there is no conflict of interest and they are not currently working with the family in another setting.

2. Ask if the interpreter has experience of working in a CAMHS setting. They may need additional training.

3. Ask if the interpreter has experience of interpreting for deaf children using various communication modalities. Do they have knowledge of deaf children's language development? Do you need this information? Do you need the services of a deaf interpreter?

4. Check the interpreter has supervision arrangements in place, if not is this something that you/your service can provide?

5. Arrange a meeting with the interpreter, before a course of therapeutic sessions, to discuss how you will work together, and the particular therapy to be used.

6. Remember to schedule additional time for the appointment; include pre and post briefing with the interpreter.

7. Consider how you will contact families, for example are letters written in plain English or translated into BSL on DVD's, can you send text messages? Can you source someone to help with this you may need to discuss filming the interpreter translating/interpreting after a session (do you need to check your services information governance in regards to these?) 


\section{References}

Ackroyd, V., Wright, B. (2018). Working with British Sign Language (BSL) interpreters: lessons from child and adolescent mental health services in the UK. Journal of Communication in Healthcare, 11(3), 195204.

Andrés-Roqueta, C., Adrian, J. E., Clemente, R. A., \& Katsos, N. (2013). Which are the best predictors of theory of mind delay in children with specific language impairment? International Journal of Language \& Communication Disorders, 48(6), 726-737.

Beresford, B., Greco, V., Clarke, S., \& Sutherland, $H$. (2008). An evaluation of specialist mental health services for deaf children and young people. York: Social Policy Research Unit, University of York.

Brenman, N. F., Hiddinga, A., Wright, B. (2017). Intersecting cultures in deaf mental health: An ethnographic study of NHS professionals diagnosing autism in D/deaf children. Culture, Medicine, and Psychiatry, 41(3), 431-452.

Emond, A., Ridd, M., Sutherland, H., Allsop, L., Alexander, A., \& Kyle, J. (2015). Access to primary care affects the health of Deaf people. BrJ Gen Pract, 65(631), 95-96.

Fonagy, P., Campbell, C., \& Luyten, P. (2017). Mentalizing. In S.N. Gold (Ed) APA Handbooks in Psychology. APA Handbook of Trauma Psychology: Foundation of knowledge (p373 - 383). American
Psychological Association.

Luke, N., \& Banerjee, R. (2013). Differentiated associations between childhood maltreatment experiences and social understanding: A metaanalysis and systematic review. Developmental Review, 33(1), 1-28.

Peterson, C. C. (2016). Empathy and theory of mind in deaf and hearing children. Journal of deaf studies and deaf education, 21(2), 141-147.

Schick, B., De Villiers, P., De Villiers, J., \& Hoffmeister, R. (2007). Language and theory of mind: A study of deaf children. Child Development, 78(2), 376-396.

Wright, B., \& Oakes, P. (2012). Does Socio-emotional Developmental Delay Masquerade as Autism in Some Deaf Children?. International Journal on Mental Health and Deafness, 2(1).

Wright, B., Walker, R., Holwell, A., Gentili, N., Barker, M., Rhys-Jones, S., ... \& Moore, K. (2012). A new dedicated mental health service for deaf children and adolescents. Advances in Mental Health, 11(1), 95-105.

Young, A., Ferguson-Coleman, E., Wright, B., Le Couteur, A. (2019). Parental Conceptualizations of Autism and Deafness in British Deaf Children. The Journal of Deaf Studies and Deaf Education, 24(3), 280-288. 


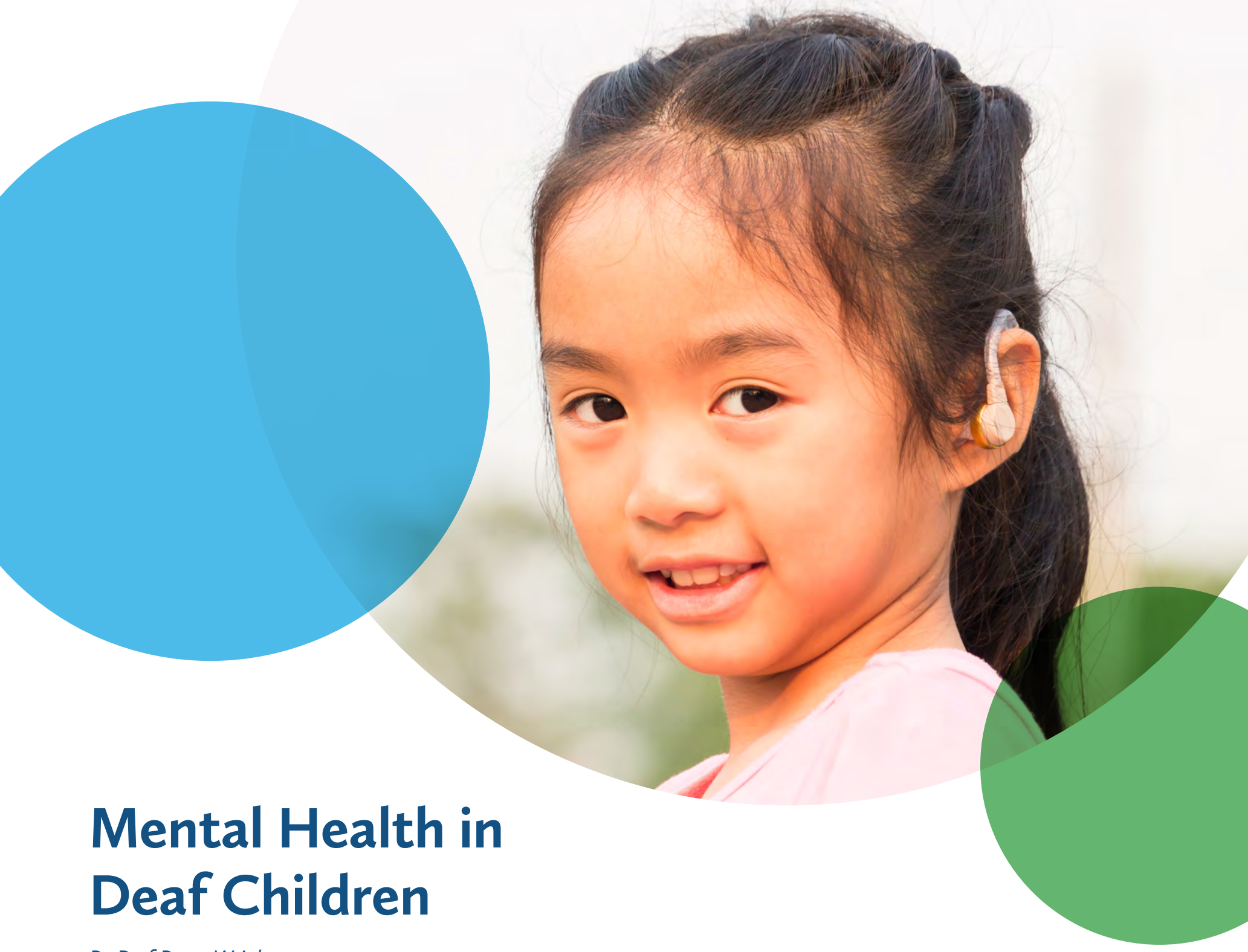

By Prof Barry Wright

The most recent study of mental health in deaf children in England showed that $26 \%$ in a sample of 144 signing deaf children and young people not currently accessing child mental health services had a probable mental health problem and $57 \%$ had a possible mental health problem (Roberts et al, 2015). This is much higher than the general population of 11-16 year olds (Sadler et al, 2018) where approximately $14 \%$ are thought to have a mental disorder. Developing an understanding or a model for why these rates are so much higher would be very helpful for two main reasons. The first is specific to developing an understanding of the needs of the deaf child population so that we can begin to think more about service planning and particularly understanding social, emotional and mental health needs. The second is that when we consider the stressors that different groups of deaf children are placed under and why it is that deaf children have higher rates of mental health problems it may give us a much richer understanding of the multiple routes to mental health problems.

What is it like to be a deaf child in a hearing community? We know from research that deaf children are more likely to be isolated, bullied or abused (Wolters et al, 2011; Kvam, 2004), which can impact upon mental health. There is a large literature on prejudice towards deaf children and their families and experiences of feeling stigmatised.

Over $90 \%$ of deaf children are born within hearing families and most of them are not expecting a deaf child. This leads to difficulties in adjusting to the new challenges of parenting and educational choices and can be complicated by very different views and/or advice about what the best approach to take is. Parents are almost always dedicated to their children, but may also struggle with anxiety, depression, over-protection, challenges with relationship and attachment, and rarely rejection of their child. All of these have mental health consequences for the child.

We also know that the government's inclusion policy means that most deaf children are in mainstream schools and they are often the only deaf child in their class. This makes the likelihood of them having a deaf peer group in any meaningful day to day way very limited. It also means that large numbers of mainstream 
teachers, often with limited training have to make sure that the curriculum is available to those children. A large proportion of deaf children have single gene causes for being deaf and no intellectual, neurological or congenital problems but these children do not do as well as hearing children at all examination test stages (Hendar \& O'Neill, 2016) suggesting that the education system may struggle to meet fully the needs of many deaf children.

Another factor relates to communication. There is a tendency for the research literature to consider all deaf children together as if they are the same. In fact, they all have very different life trajectories with different causes for being deaf, occurring at different times, in different families who make different and sometimes changing educational and communication choices. Some deaf children communicate through spoken English, especially those who are mildly deaf. Some children communicate completely in British Sign Language (BSL) with no spoken English. BSL is a fully recognised UK language, but despite this it is not always readily available in our education or workplace systems. BSL has a different word order, grammar and syntax to spoken English and does not map easily on to spoken or written English. The UK system is not well prepared for this variability. This creates a number of challenges for deaf children and their families that include high levels of stress, communication problems and social and emotional challenges. Some children are being educated in bilingual ways and are being taught spoken and written English as well as BSL or sign supported English (using the English word order with adjunctive signs). Attempting to learn multiple communication modalities can be very challenging for children, especially for that subgroup of deaf children with additional complex problems or intellectual disabilities. This can create its own problems. Deaf children sometimes have less exposure to language learning and may have language deprivation and/or delay, which can in turn lead to delays in theory of mind (empathy-related) skills (Peterson et al, 2016) compounding problems in social settings.

Some deaf children may be deaf as a result of a neurological insult or a more complex genetic problem that leads to multiple difficulties including intellectual disability and/or a range of possible physical problems such as visual problems, balance problems or other organ damage (e.g. heart, kidney etc.) (Van Dijk et al, 2010). These all carry their own increased mental health risks.

This landscape helps us understand the multiple myriad pathways to mental health problems. This population of young people has much to commend it. Meeting deaf children and young people it is clear to see their joys and enthusiasms, their hopes for the future and their numerous strengths and abilities, as well as the multiple challenges that they face. It would be instructive to understand this group better in order to be able to provide better community based services, education services, health services and mental health services for deaf children and their families, but also as a learning experience to help us as professionals to develop innovative therapeutic strategies that can engage with the multiple pathways to mental health problems.

\section{References:}

Hendar, O. and O'Neill, R., 2016. Monitoring the achievement of deaf pupils in Sweden and Scotland: Approaches and outcomes. Deafness \& education international, 18(1), pp.47-56.

Kvam, M. H. (2004). Sexual abuse of deaf children. A retrospective analysis of the prevalence and characteristics of childhood sexual abuse among deaf adults in Norway. Child Abuse \& Neglect, 28(3), 241-251.

Peterson, C.C., O'Reilly, K. and Wellman, H.M., 2016. Deaf and hearing children's development of theory of mind, peer popularity, and leadership during middle childhood. Journal of experimental child psychology, 149, pp.146-158.

Roberts, S., Wright, B., Moore, K., Smith, J., Allgar, V., Tennant, A., Doherty, C., Hughes, E., Moore, D.C., Ogden, R. and Phillips, H., 2015. Translation into British Sign Language and validation of the strengths and difficulties questionnaire. Health Technology Assessment Report. Feb 2015 s(2) National Institute for Health Research Journals Library: Southampton.

Sadler, K., Vizard, T., Ford, T., Marchesell, F., Pearce, N., Mandalia, D., Davis, J., Brodie, E., Forbes, N., Goodman, A. and Goodman, R., 2018. Mental health of children and young people in England, 2017.

van Dijk, R., Nelson, C., Postma, A. and van Dijk, J., 2010. Deaf children with severe multiple disabilities: Etiologies, intervention, and assessment. Oxford handbook of deaf studies, language, and education, 2, pp.171-192.

Wolters, N., Knoors, H.E., Cillessen, A.H. and Verhoeven, L., 2011. Predicting acceptance and popularity in early adolescence as a function of hearing status, gender, and educational setting. Research in developmental disabilities, 32(6), pp.25532565. 



\title{
. (3) . \\ Do audiological classification systems cause more confusion than clarity?
}

\author{
By Dr. Jessica K Edwards
}

In 2018, Barry Wright and colleagues at the Lime Trees Child, Adolescent and Family Unit in York published a paper in the International Journal on Mental Health and Deafness that highlighted the wide variation between classification systems for hearing loss. In this enlightening piece, Wright et al. show that six of the most commonly used classification systems all assign the same descriptive terms to different decibel ( $\mathrm{dBHL}$ ) thresholds (Table 1). For example, there is a staggering $15 \mathrm{dBHL}$ difference in the definition of "profound" deafness between the World Health Organisation's classification system ( $\geq 81)$ and the American Medical Association's system $(\geq 96 \mathrm{dBHL})$. The lack of a single classification system is clearly problematic, not least because it renders intervention studies difficult to interpret and has implications for patient access to services. Going forward, Wright et al. hope that awareness of the differences between these classification systems will improve. Meanwhile work is needed to determine whether a unified system might be helpful. Finally, they explain that practitioners should ideally consider audiological thresholds, as just one part of a holistic assessment, of sensory profiles and quality of life.

\section{Table 1: Different systems of classification}

Reproduced with permission from Wright, B. et al. (2018), The use of audiological classification systems, International Journal on Mental Health and Deafness. 4: 59-64.

\begin{tabular}{|c|c|c|c|c|c|}
\hline Units = db & Mild & Moderate & $\begin{array}{l}\text { Moderate } \\
\text { to Severe }\end{array}$ & Severe & Profound \\
\hline American Medical Association * & $26-40$ & $41-70$ & & $71-95$ & $\geq 96$ \\
\hline $\begin{array}{l}\text { American Speech Language } \\
\text { Hearing Association }\end{array}$ & $26-40$ & $41-55$ & $56-70$ & $71-90$ & $\geq 91$ \\
\hline British Society of Audiology * & $20-40$ & $41-70$ & & $71-95$ & $\geq 96$ \\
\hline $\begin{array}{l}\text { Center for Disease Control } \\
\text { hearing loss criteria for } 2009 \text { and } \\
2010 \text { surveys }\end{array}$ & $21-40$ & $41-70$ & & $71-90$ & $\geq 91$ \\
\hline $\begin{array}{l}\text { Prevention Atlanta } \\
\text { Developmental Disabilities } \\
\text { Surveillance Programme 灹 }\end{array}$ & & $40-64$ & & $65-84$ & $\geq 85$ \\
\hline World Health Organisation $* * * *$ & $26-40$ & $41-60$ & & $61-80$ & $\geq 81$ \\
\hline
\end{tabular}

* Taken in the better ear over 5 frequencies (0.25, 0.5, 1, 2 \& 4 kHertz)

$* *$ Taken as better ear average over 3 frequencies (0.5, 1, 2 Khertz)

**** Taken as better ear average over 4 frequencies (0.5, 1, 2, 4 KHertz) 


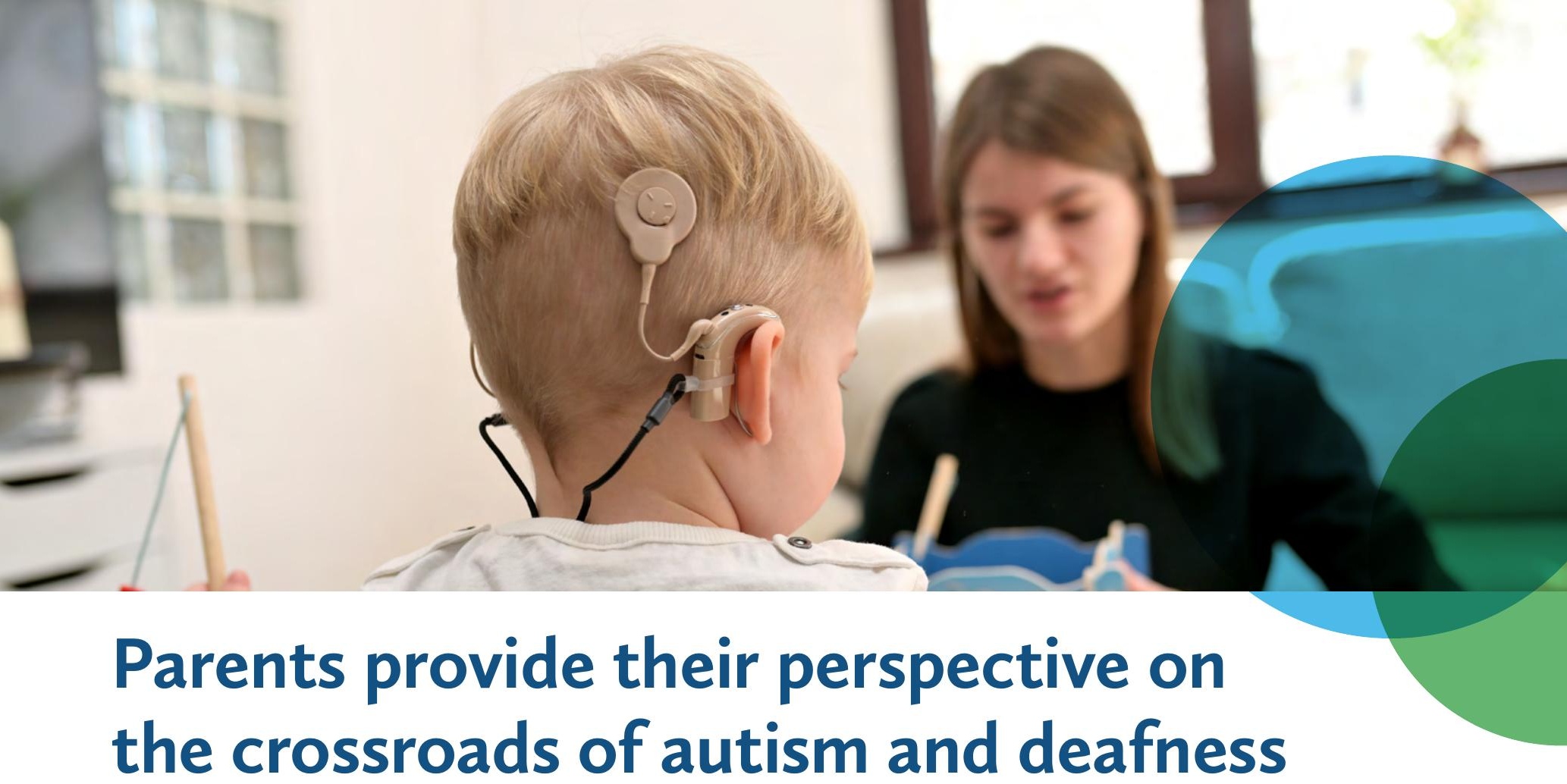

By Dr. Jessica K Edwards

Deafness and autism share many developmental features in affected children, including language delays, problems with specific language functions, pragmatic language difficulties and delayed theory of mind. Unsurprisingly, "diagnostic overshadowing" - whereby autism might mask hearing loss or intellectual disability, and vice versa - is a real concern in children affected by both conditions. ${ }^{2}$

In 2019, Alys Young and colleagues asked eight parents to give their perceptions on the interaction between deafness and autism in their child. Some parents said that practitioners had made false attributions about their child's behaviours and what is assumed to be normal behaviour for a deaf child. Others said that comparisons were made between their child's development and the typical trajectories of development for a deaf child. While sometimes useful, Young et al. explain that this comparison could mask other underlying medical or genetic conditions. Finally, some parents reported that deafness was not relevant to them when initially considering that their child might have autism.

The researchers also asked the parents how a child, who experiences deafness and autism, affects their family's everyday life. A few key points were raised, including: that autism has more of an effect on some children than deafness, that autism affects sign language development, that deafness can interfere with sound and sensory stimulation, and that working out what behaviours might be due to deafness and what might be due to autism is difficult.

Although only eight parents were interviewed, the insights gained from these interviews evoke many implications for clinical practice. For example, Young et al. explain that clinicians should have a firm understanding of typical deaf child development, to ensure that false attributions are not made. When devising diagnostic/support pathways, clinicians should also consider how hearing and deaf children interact with their environment. Perhaps most importantly, clinicians must recognize that the interaction between autism and deafness is dynamic: how a parent conceptualizes this interaction is important, when tailoring support packages to affected families.

\section{Referring to:}

Young, A., Ferguson-Coleman, E., Wright, B. \& Le Couteur, A. (2019), Parental conceptualizations of autism and deafness in British deaf children. J. Deaf Stud. Deaf Educ. 24: 280-288. doi: 10.1093/ deafed/enzoo2.

\section{References:}

${ }^{1}$ Szarkowski, A. et al. (2014), A summary of current understanding regarding children with autism spectrum disorder who are deaf or hard of hearing. Semin. Speech Lang. 35: 241-259. doi:10.1055/s-0034-1389097.

${ }^{2}$ Wright, B. et al. (2012), Does socio-emotional developmental delay masquerade as autism in some deaf children? International Journal of Mental Health and Deafness, 2: 45-51.

\section{Glossary:}

Theory of mind: a social-cognitive skill to attribute mental states to oneself and to other people so as to interpret, explain and predict behaviour. 


\title{
Integrating interpreters into CAMHS: useful tips for effective co-working
}

\author{
By Dr. Jessica K Edwards
}

Vicci Ackroyd and Barry Wright have put together a useful set of principals by which British Sign Language (BSL) interpreters, and child and adolescent mental health services (CAMHS) can effectively co-work with each other. Published in the Journal of Community Healthcare, Ackroyd and Wright explain that BSL interpreters form a crucial component of the multi-disciplinary therapeutic team. Indeed, a CAMHS audit in 20121 found that BSL interpreters predominantly work side-by-side with clinicians and contribute to team meetings (Figure $\mathbf{1}$ ). Ackroyd et al. highlight how essential it is that interpreters work flexibly to accommodate various communication styles (taking into account cultural and linguistic differences) and explain any communication difficulties to the clinician. In addition, both the interpreter and the clinician should reassure children and their families about patient confidentiality, and allow adequate time after a session to debrief. Going forward, Ackroyd and Wright highlight the importance of interpretation being of a high standard and that interpreters are respectful of the independence and autonomy of the deaf person and have experience of working within CAMHS.

\section{Referring to:}

Ackroyd, V. \& Wright, B. (2018), Working with British Sign Language (BSL) interpreters: lessons from child and adolescent mental health services in the U.K. J. Commun. Healthc. 11: 195-204. doi: $10.1080 / 17538068.2018 .1492218$.

\section{References:}

${ }^{1}$ Knight A. Northern National Deaf CAMHS: Team Interpreter Audit, Results and Recommendations. York: National Deaf CAMHS (Northern Arm); March 2012.

Figure 1: Percentage of time team interpreters spent by activity Reproduced with permission from Ackroyd, V. \& Wright, B. (2018), Working with British Sign Language (BSL) interpreters: lessons from child and adolescent mental health services in the U.K. J. Commun. Healthc. 11: 195-204. doi: 10.1080/17538068.2018.1492218.

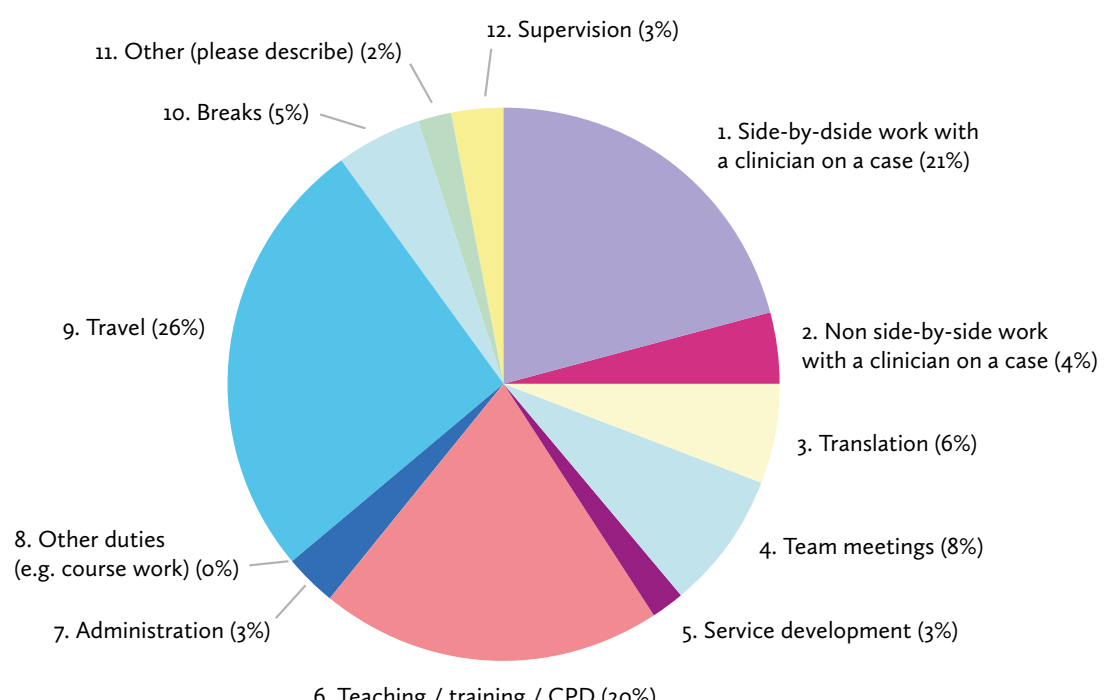

6. Teaching / training / CPD (20\%)

Figure 1. Percentage of time team interpreters spent by activity (Time 2). 


\title{
Multi-disciplinary teams are needed to sensitively diagnose autism in Deaf children
}

\author{
By Dr. Jessica K Edwards
}

An ethnographic study of NHS professionals, who diagnose autism in Deaf children, finds that recognizing the intersections between mental health and Deaf culture is essential for healthcare professionals to make sensitive diagnoses. The study, conducted by researchers in the UK and the Netherlands and published in the journal of Culture, Medicine and Psychiatry was based on an analysis of the practices and perceptions of 16 health service professionals who have conducted autism assessments for Deaf children aged between 0 and 18 years.

Natassia Brenman and colleagues learnt that professionals working in specialist Deaf Services, or with experience working with the Deaf community, had an intersectional understanding of autism assessments. As such, these professionals used their knowledge of how cultural, linguistic, sensory, and social factors interact when making their diagnoses. Many professionals highlighted that Deaf language and culture can be markedly different from social communication observed in the hearing population. Consequently, a commonly vocalized frustration was the emphasis on using a diagnostic system that focuses on comparisons to 'norms' based on populations from a hearing culture.

Undertaking an autism assessment is more complex when the affected child is Deaf. Indeed, the primary issue raised by the professionals involved in this study was how to differentiate between the features or behaviours they should attribute to autism, and which to deafness. Going forward, Brenman and colleagues posit that multi-disciplinary diagnostic teams are necessary to help recognize the various dimensions of a Deaf child's experience and abilities.

\section{Referring to:}

Brenman, N.F., Hiddinga, A. \& Wright, B. (2017), Intersecting cultures in deaf mental health: an ethnographic study of NHS professionals diagnosing autism in D/deaf children. Cult. Med. Psychiatry. 41: 431-452. doi: 10.1007/s11013-0179526-y.

\section{Glossary:}

Intersectionality: a theory that suggests that a person who has numerous identities will have different life experiences (cultural, social and clinical) than a person who shares only one or some of those identities. 